\title{
Evidence of the Involvement of Spinal EZH2 in the Development of Bone Cancer Pain in Rats
}

\author{
Haoming Chen* \\ Jianmang $\mathrm{Yu}^{*}$ \\ Lihua Hang* \\ Shuai Li \\ Weikang Lu \\ Zhenkai Xu
}

Department of Anesthesiology, Kunshan First People's Hospital Affiliated to Jiangsu University, Kunshan, Jiangsu, 215300, People's Republic of China

*These authors contributed equally to this work
Correspondence: Lihua Hang Department of Anesthesiology, Kunshan First People's Hospital Affiliated to Jiangsu University, Suzhou, Jiangsu Province,

Peoples Republic of China

Tel +86-18013273169

Email zjhanglihua@foxmail.com
Introduction: Bone cancer pain (BCP) seriously affects the quality of life of patients with advanced cancer, but effective treatment methods are lacking. This study mainly investigates the role of EZH2 in a well-established BCP model induced by Walker 256 breast cancer cells in rats.

Methods: Female Sprague-Dawley rats of the same age weighing approximately 160 $\mathrm{g}$ were selected for the experiment. The BCP model was established by injecting inactivated Walker 256 breast cancer cells into the tibia. von Frey filaments were used to measure the paw withdrawal threshold, and bone destruction in the rat was observed using x-ray. The spinal EZH2 and H3K27Tm levels were measured using Western blotting and RT-qPCR analysis. Intrathecal injection of an $\mathrm{EZH} 2$ inhibitor was performed to examine the role of EZH2 in trigeminal BCP.

Results: Experimental results showed that injecting Walker 256 breast cancer cells into the tibia induced bone cancer pain. Spinal EZH2 and H3K27Tm levels were significantly increased over time in BCP rats. An intrathecal injection of 3-deazaneplanocin A (DZNep), a selective EZH2 inhibitor, downregulated the expression of EZH2 and attenuate the BCP-induced mechanical allodynia state.

Conclusion: Intrathecal injection of DZNep relieve bone cancer pain in rats. EZH2 expressed in spinal cord tissue may be involved in the process of bone cancer pain in rats. Keywords: enhancer of zeste homologue 2, bone cancer pain, histone methyltransferase, 3 deazaneplanocin $\mathrm{A}$, intrathecal injection

\section{Introduction}

Cancer-related pain is the main factor contributing to a poor quality of life of patients. Based on related studies, bone is the most vulnerable tissue to cancer metastasis and readily experiences pain. ${ }^{1,2}$ In addition, bone cancer pain (BCP) is the most severe and uncontrollable pain compared with other types of cancer pain. Currently, the molecular mechanism of BCP remains unclear, and suitable therapeutic drugs are lacking. Common clinical therapeutic drugs for BCP cannot be used in large quantities or long-term due to serious adverse reactions and addiction. ${ }^{3}$ Therefore, a better understanding of the molecular mechanism of BCP is necessary.

Zeste gene enhancer homologue $2(\mathrm{EZH} 2)$ is human homologue 2 of the Drosophila zeste gene enhancer. ${ }^{4}$ The structure of EZH2 is complex and mainly includes the following four highly conserved domains: I, II and cysteine regions and the SET domain composed of the Su3-9, enhancer of zeste and trithorax. ${ }^{5}$ EZH2 is an important member of the polycomb-group (PcG) protein family. ${ }^{6}$ The PcG family 
includes two complexes, PRC1 and PRC2, which maintain gene suppression and gene silencing, respectively. ${ }^{7}$ PRC2 mainly methylates lysines 9 and 27 of histone 3 through the SET domain of EZH2, thereby silencing a series of downstream target genes, including genes involved in cell differentiation, pain and a series of related genes. Therefore, $\mathrm{EZH} 2$ is one of the three subunits comprising PRC2.

Based on accumulating evidence, levels of EZH2 and trimethylation of lysine 27 in histone 3 (H3K27 trimethylation, $\mathrm{H} 3 \mathrm{~K} 27 \mathrm{Tm}$ ) are increased in the spinal dorsal horn of rats with neuropathic pain. Intrathecal injection of DZNep, an inhibitor of EZH2, attenuates the development of neuropathic pain. ${ }^{8}$ These findings indicated that EZH2 may be involved in neuropathic pain. However, BCP is not completely equivalent to neuropathic pain. BCP includes some common characteristics of inflammatory pain and neuropathic pain, as well as its own characteristics. ${ }^{9}$ At present, few studies have assessed whether $\mathrm{EZH} 2$ is involved in the process of BCP. In this study, we verified the important role of $\mathrm{EZH} 2$ in the spinal cord of BCP rats and provided a possible theoretical basis for the development of new drugs to treat BCP.

\section{Materials and Methods}

\section{Animals}

Eighty-eight female Sprague-Dawley rats weighing 160 to $180 \mathrm{~g}$ were obtained from the Zhao Yan Limited Research Center in Suzhou, China. Before starting the experiment, all animals were acclimated to the environment for 3 days, housed at a temperature of approximately $21 \pm 3{ }^{\circ} \mathrm{C}$, and maintained on a photoperiod of 07:00-19:00, with food freely available. The animal experiments were approved by the Institutional Animal Care and Use Committee of Jiangsu University (DJS-LAER-AP-2018030635) and performed in accordance with the National Institutes of Health Guide for Care and Use of Laboratory Animals (Publication No. 80-23, revised 1996).

\section{Bone Cancer Pain Model}

Walker 256 breast cancer cells were kindly provided by Soochow University, and the use of the cell line was approved by the Institutional Animal Care and Use Committee of Jiangsu University and the Ethics Committee of Kunshan First People's Hospital. Rats were anaesthetized with an intraperitoneal injection of $5 \%$ chloral hydrate $(400 \mathrm{mg} / \mathrm{kg})$. The skin was cut longitudinally at the middle and lower $1 / 3$ of the left tibia to expose the tibia, and a $25 \mathrm{G}$ sterile needle attached to a syringe was used to drill a hole perpendicular to the tibia. ${ }^{10}$ The bone marrow cavity of rats in the BCP group was injected with Walker 256 cells (approximately $1 \times 10^{5}$ cells, $10 \mu \mathrm{L}$ ), and rats in the sham group were injected with heat-killed Walker 256 cells. Cells were injected slowly, the tibial canal was sealed with bone wax, and the wound was sealed with tissue glue to prevent leakage of cells outside the bone. ${ }^{11}$ Rats with obvious swelling and wound redness were excluded.

\section{Intrathecal Catheterization}

Rats were anaesthetized with an intraperitoneal injection of $5 \%$ chloral hydrate $(400 \mathrm{mg} / \mathrm{kg})$. A longitudinal incision approximately $1 \mathrm{~cm}$ in length was made at L4-5 of the spine. After puncturing the ligamentum flavum and dura mater with a $25 \mathrm{G}$ needle attached to a syringe, clear cerebrospinal fluid flowed out. The PE capillary catheter $(1.5 \mathrm{~cm})$ was inserted through the ruptured spinal membrane and then fixed at the back of the neck. ${ }^{12}$ Finally, the incision was sutured carefully. After an intrathecal injection of $10 \mu \mathrm{L}$ of $1 \%$ lidocaine, paralysis of the lower limbs on both sides of the rat confirmed the successful catheterization. Rats with nerve injury, paralysis, and poor wound healing were excluded. Usually, the intrathecal dose was $10 \mu \mathrm{L}$, followed by $10 \mu \mathrm{L}$ of saline to flush the tube. ${ }^{13}$

\section{Pain Behaviour Analysis}

We measured the ambulatory pain score (AS) and paw withdrawal threshold (PWT) of eight rats in each group to assess changes in behaviour before and after inoculation. Briefly, on the day of the measurement, the rats were placed in a transparent Plexiglas box $(15 \times 15 \times 30 \mathrm{~cm})$, and the box was placed on a metal mesh frame. The rats could walk freely in the box. After 30 minutes of acclimation, the score was recorded based on the degree of lameness observed during walking. 0 point: rats can move freely and walk normally, 1 point: rats walking speed decreased but could still walk normally, 2 points: obvious discomfort with a degree ranging from 1-3 points, 3 points: rats were severely restricted in walking and the walking time is significantly reduced, and 4 points: the rat's hind limbs did not touch the ground. Scores were recorded based on these criteria, and finally the AS was calculated from the values of three consecutive tests separated by intervals of $30 \mathrm{~s} .{ }^{14}$

For the PWT measurement, rats were allowed to acclimate for 30 minutes before the experiment. The calibrated von Frey filaments were applied perpendicularly to the left plantar 
posterior of the rat. The PWT was determined by the size of von Frey filaments applied when the rat quickly retracted or raised the leg. The mean PWT was calculated from the values of three consecutive tests separated by intervals of $60 \mathrm{~s} .{ }^{15}$

\section{X-Ray Detection of Bone}

After the administration of $5 \%$ chloral hydrate $(400 \mathrm{mg} / \mathrm{kg}$ ) anaesthesia, the rats were placed in the prone position and an X-ray was captured ( $47 \mathrm{Kv}, 0.5 \mathrm{~mA}$, Siemens, Germany).

\section{Real-Time Quantitative PCR and Western Blotting}

Rats were anaesthetized with an intraperitoneal injection of $5 \%$ chloral hydrate $(400 \mathrm{mg} / \mathrm{kg})$. Spinal L4-6 tissues were dissected from rats on ice and stored at $-80{ }^{\circ} \mathrm{C}$ until use. Spinal cDNAs were fully obtained through reverse transcription. The expression of the EZH2 mRNA relative to $\beta$-actin was calculated using the $2^{-\Delta \Delta} \mathrm{Ct}$ method.

For Western blot analysis, $50 \mu \mathrm{g}$ of protein from the spinal homogenate was obtained, separated on an SDS-PAGE gel and transferred to a PVDF membrane. Membranes were blocked with 5\% non-fat milk for at least $1 \mathrm{~h}$ and incubated with polyclonal rabbit anti-EZH2 (1:500, Abcam), antiH3K27Tm (1:500, Abcam) or anti- $\beta$-actin (1:2000, Abcam) primary antibodies overnight at $4{ }^{\circ} \mathrm{C}$; an HRP-conjugated goat antirabbit IgG H\&L (1:5000, Abcam) secondary antibody was subsequently incubated with the membrane. The resulting bands were analysed using ImageJ software.

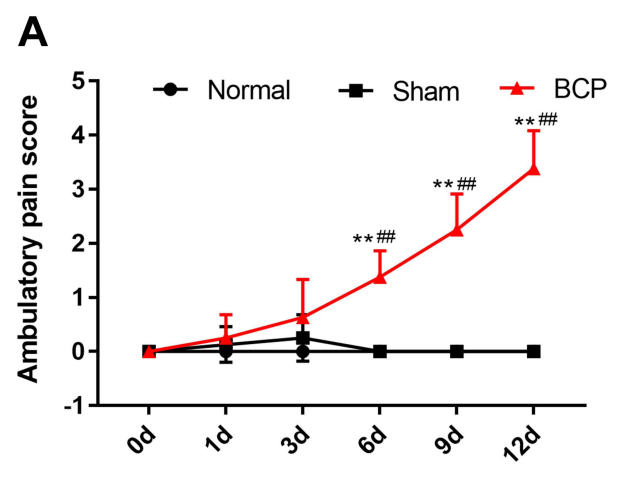

Days after Walker 256 inoculation

\section{Statistical Analysis}

All data are presented as the means \pm SEM and were analysed using SPSS 21.0 software. Two-way ANOVA followed by a Bonferroni post hoc analysis was used to analyse the behavioural data. One-way ANOVA was used to analyse qPCR and Western blot data. P values less than 0.05 were considered statistically significant.

\section{Results}

\section{Inoculation of Walker 256 Cells into the Tibia Induced Hyperalgesia in Rats}

During the 12-day observation period, the rats were in good health. No significant difference in the baseline PWT was observed between rats in the normal group and the sham group. The AS value of rats in the BCP group increased gradually from the normal level of $0 \pm 00$ to 3.38 \pm 0.70 on the 12 th day (Figure $1 \mathrm{~A}, \mathrm{~F} 23=81.0, \mathrm{~N}=8$, $P<0.01$ ), and the PWT value decreased from the normal level of $34.00 \pm 4.00$ to $6.25 \pm 1.56$ on the 12th day (Figure 1B, F23=170.0, $\mathrm{N}=8, P<0.01$, $\underline{\mathrm{S}-\text { Table 1) }}$.

\section{Bone X-Ray}

$\mathrm{X}$-rays of the normal group and sham group showed that the left tibia bone was not damaged, the cortical bone was smooth, and no bone tumour growth occurred. On the 6th day after inoculation in the BCP group, the tibial bone density began to decrease, and the bone of the epiphysis appeared to be slightly damaged. Bone density

B

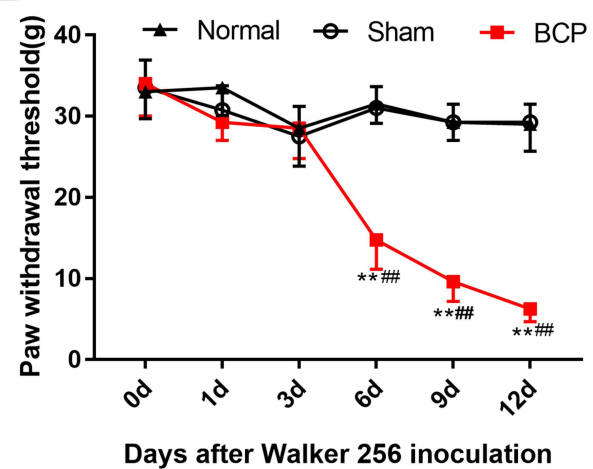

Figure I Inoculation of Walker 256 cells into the tibia induced hyperalgesia in rats. During the I2-day observation period, (A) the AS value of the BCP group gradually increased compared with the normal group and sham group. (B) The PWT value gradually decreased compared with the normal group and sham group. Data are presented as the means \pm SEM for 8 rats. Compared with the normal group, ${ }^{*} * P<0.01$; compared with the sham group, ${ }^{\#} P<0.01$.

Abbreviations: AS, ambulatory pain score; PWT, paw withdrawal threshold; BCP, bone cancer pain. 
was further reduced, and the range of bone destruction increased on the 9th day. Finally, the cortical bone morphology began to change on the 12th day (Figure 2).

\section{Increased Spinal EZH2 and H3K27Tm Levels in BCP Rats}

Compared with the normal group and the sham group, the qPCR results showed significantly increased expression of the EZH2 mRNA on Days 6, 9, and 12 in BCP rats, and the highest expression level was detected on the 6th day (Figure 3A, F11=140.3, N=4, $P<0.01$ ). Western blot results showed significantly higher levels of the EZH2 and $\mathrm{H} 3 \mathrm{~K} 27 \mathrm{Tm}$ proteins in the BCP group than those in the normal and sham groups, and the highest levels were observed on the 9th day (Figures 3B and C). These results suggest that increased levels of EZH2 and HK27Tm may contribute to $\mathrm{BCP}$ in rats.

\section{Intrathecal Injection of the EZH2 Inhibitor DZNep Relieved BCP and Reduced EZH2 Expression}

The EZH2 inhibitor DZNep (20 nM, Selleck, USA) was injected intrathecally into BCP rats from Day 3 to Day 5 (once a day for 3 days) after inoculation to further study the role of EZH2 in the spines of BCP rats. The AS and PWT of rats in the $\mathrm{BCP}+\mathrm{DZNep}$ group improved from Days 6 to 12 after inoculation (Figure $4 \mathrm{~A}$ and $\mathrm{B}$, $\mathrm{F} 31=26.97, \mathrm{~N}=8, P<0.01$ and $4 \mathrm{C}, \mathrm{F} 31=114.92, \mathrm{~N}=8$, $P<0.01$, S-Table 2). Western blot results showed significantly lower EZH2 expression in the $\mathrm{BCP}+\mathrm{DZNep}$ group than in the BCP group, but the levels were still higher than those in the sham group (Figure 4C). Thus, an intrathecal injection of DZNep relieves BCP and reduces EZH2 expression in rats.

\section{Discussion}

A classic BCP model was established by administering an intratibial injection of Walker 256 breast cancer cells. Previous studies have shown that female rats are more sensitive to pain, and thus behavioural changes are easier to observe. Furthermore, Walker 256 breast cancer cells featuring simple reproduction and strong carcinogenicity better simulate the pathophysiological process of breast cancer bone metastasis. ${ }^{16}$ Cancer cells destroy bone and produce inflammatory factors in the bone marrow cavity. ${ }^{17}$ These inflammatory factors or neuropeptides bind to the corresponding receptors on neurons and transmit
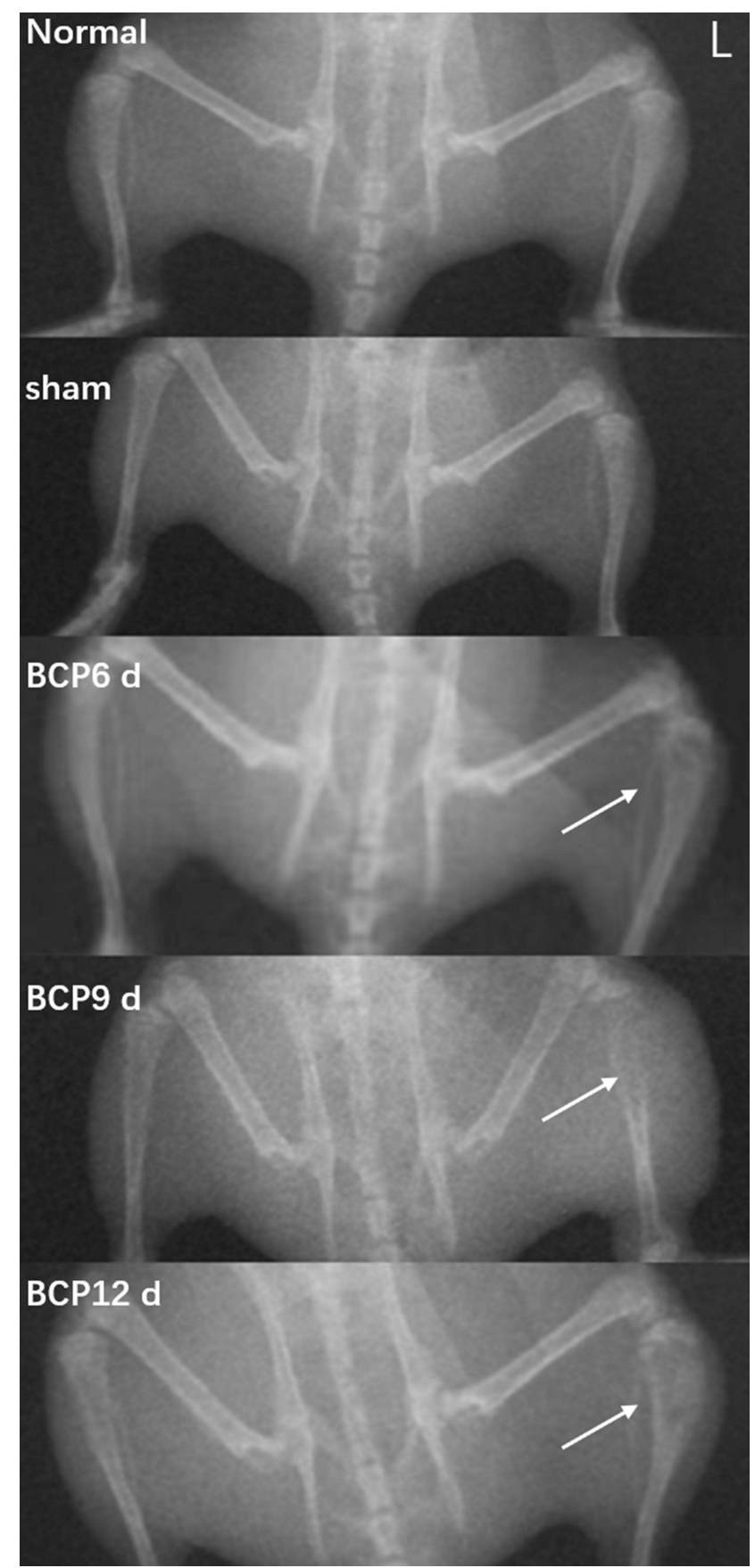

Figure 2 X-rays of the rat tibia. The bones of rats in the normal group and the sham group were not damaged. The tibial bone density began to decrease, and the bone of the epiphysis appeared to be slightly damaged on Day 6 after inoculation. Bone density was further reduced, and the range of bone destruction increased on Day 9 after inoculation. The cortical bone morphology began to change on Day 12 after inoculation. The arrow indicates a significant decrease in bone density and trabecular bone defects in the left tibial metaphysis of BCP rats.

nociceptive signals to the rat spinal lumbar enlargement through axonal reverse transmission, leading to the continuous excitement of spinal neurons. ${ }^{18}$ With the input of persistent pain signals, the primary afferent central 


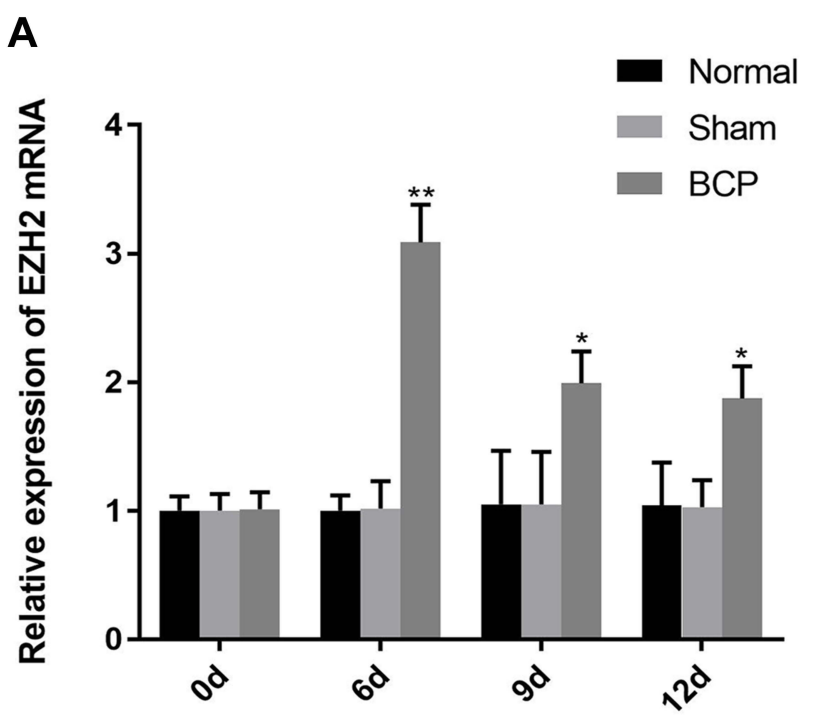

Days after Walker 256 inoculation
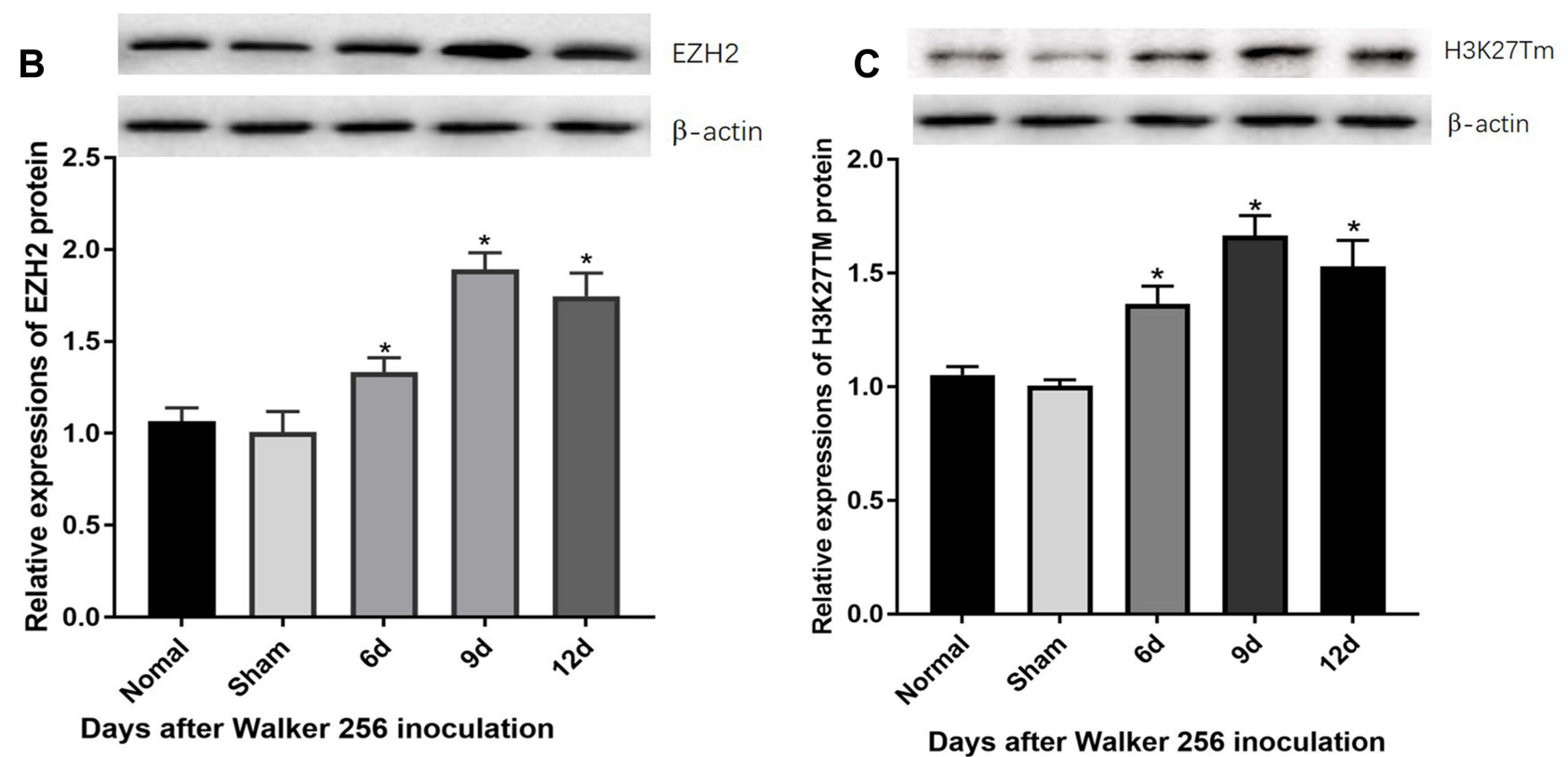

Figure 3 Increased spinal EZH2 and H3K27Tm levels in BCP rats. (A) The qPCR results showed significantly upregulated expression of the EZH2 mRNA in the spine of the BCP group. (B and $\mathbf{C}$ ) Western blot results showed significantly higher levels of the EZH2 and H3K27Tm proteins in the BCP group than those in the normal and sham groups. Data are presented as the means \pm SEM for 4 rats. Compared with the normal group, $* * P<0.01$ and $* P<0.05$.

Abbreviations: $\mathrm{EZH} 2$, enhancer of zeste homologue 2; $\mathrm{H} 3 \mathrm{~K} 27 \mathrm{Tm}$, trimethylation of lysine 27 in histone 3; BCP, bone cancer pain.

terminal releases more neurotransmitters (glutamate, CGRP, and BDNF). These neurotransmitters produce a state of overactive and overexcited neurons in the spine called central sensitization that eventually causes persistent bone cancer pain. ${ }^{19,20}$ According to the present work and previous studies, foot withdrawal and limp were observed on the 6th day after inoculation and gradually worsened, peaking on the 12th day. Therefore, we chose a 12-day observation period and completed the intrathecal injection before the 6th day.

As one of the three subunits constituting PCR2, EZH2 functions mainly by generating a trimethylation mark on H3K27 when PRC2 is recruited to chromatin, preventing RNA polymerase II-dependent transcription elongation and then silencing downstream genes. ${ }^{21,22}$ The levels of EZH2 and H3K27Tm were significantly upregulated in the 
A

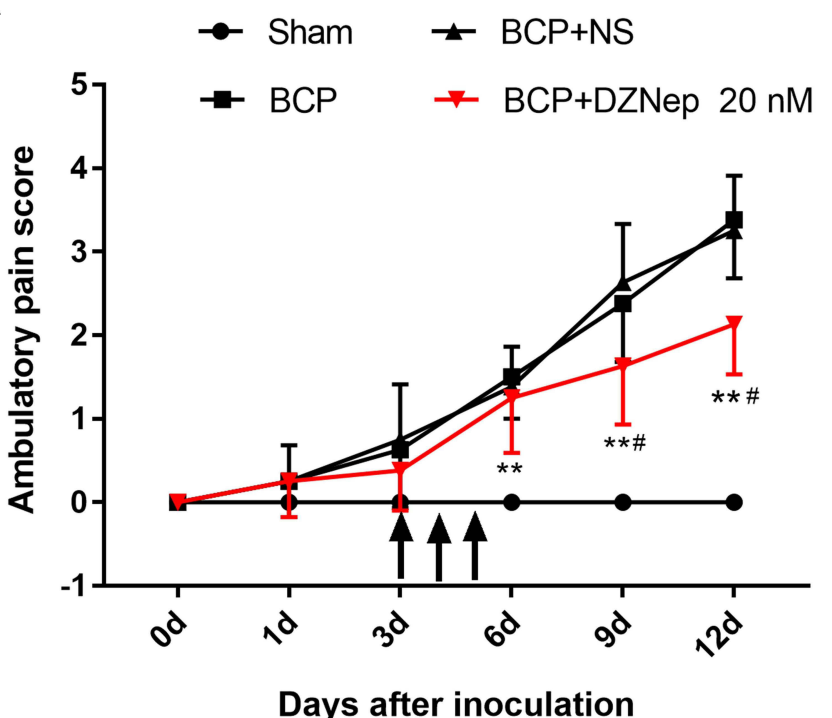

B

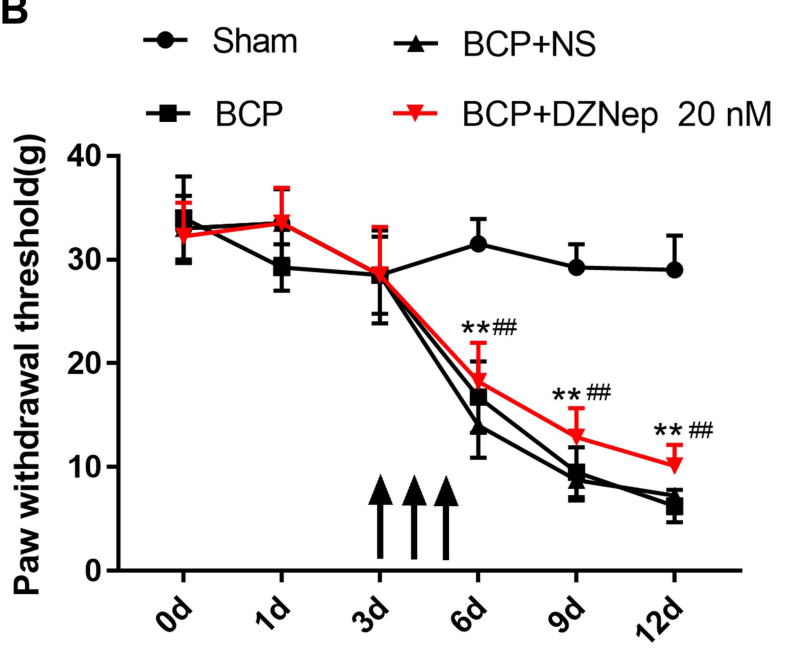

Days after Walker 256 inoculation

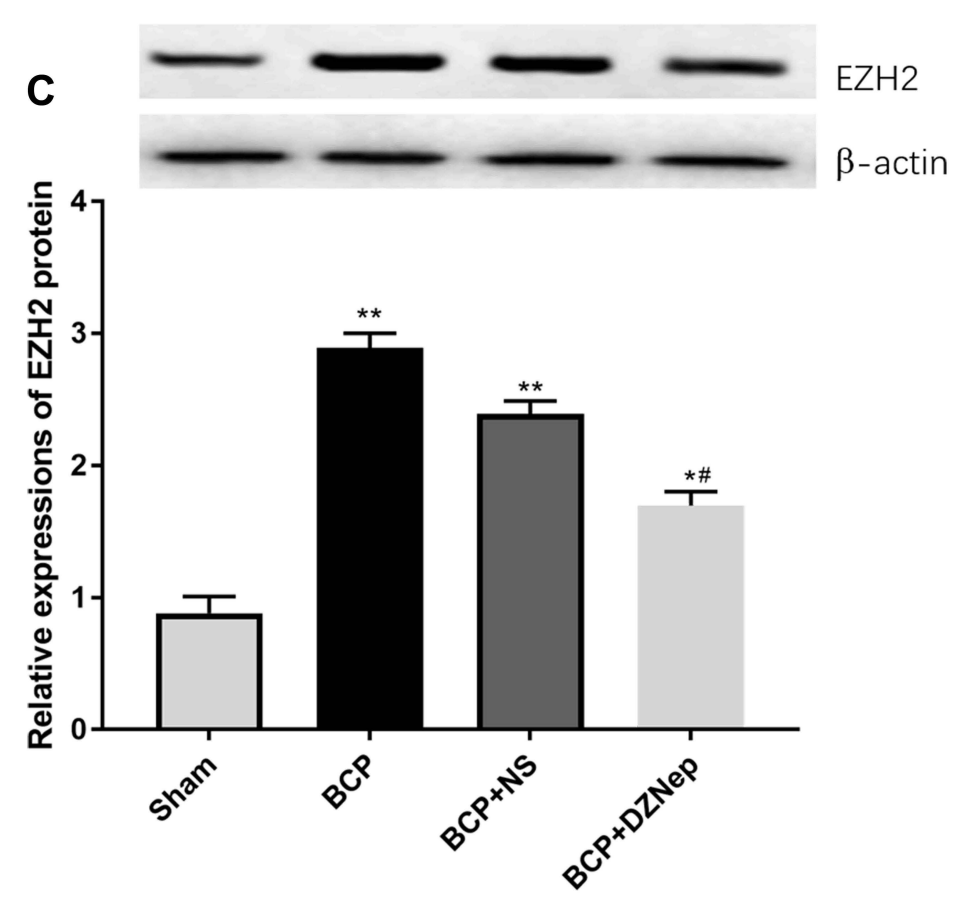

9 days after inoculation

Figure 4 Intrathecal injection of the EZH2 inhibitor DZNep relieves BCP and reduces EZH2 expression. (A and $\mathbf{B})$ The AS and PWT of rats in the BCP+DZNep group improved from 6 to 12 days after inoculation. Data are presented as the means \pm SEM for 8 rats, compared with the sham group, $* * P<0.01$, compared with the $B C P$ group, ${ }^{\# P} P<0.01$ and ${ }^{\#} P<0.05$. The arrow indicates the intrathecal injection. (C) Western blot results showed lower EZH2 expression in the BCP+DZNep group than in the BCP group, but the levels were still higher than those in the sham group. Data are presented as the means \pm SEM for 4 rats, compared with the sham group, $* * P<0.01$ and $* P<0.05$, compared with the BCP group, ${ }^{\#} P<0.05$.

Abbreviations: BCP, bone cancer pain; DZNep, 3-deazaneplanocin A.

spines of BCP rats on Days 6, 9 and 12, indicating that EZH2 may be involved in the BCP process by promoting the methylation modification. However, this experiment still has limitations due to the limited experimental conditions; for example, paraformaldehyde was not used for perfusion when the spinal cord was removed. Therefore, the results do exclude the interference of changes in the expression of the target gene in tissues such as blood, stroma, and glia. The highest expression of the EZH2 mRNA was observed on Day 6, while the highest protein expression level was detected on Day 9. The difference in mRNA and protein expression may be related to the 
mRNA secondary structure. Several studies have confirmed that a greater number of base pairs in the secondary structure of the stem increases the free energy that must be overcome by dissociation, resulting in a slower translation rate. $^{23}$ Generally, the translation process is completed within 72 hours. $^{24}$ As a result, the prolonged translation time may be due to the presence of numerous base pairs in the stem of the EZH2 mRNA. However, the specific mechanism must be investigated in future studies.

Intrathecal administration is the most suitable method to assess spine function. ${ }^{25}$ It not only directly affects spinal tissue but also has little effect on the whole body. ${ }^{26}$ DZNep is an inhibitor of S-adenosyl-L homocysteine hydrolase that decreases the expression levels of the EZH2 protein and H3K27me $3 .^{27}$ The results of preliminary studies indicate no statistically significant difference in PWT values of BCP rats intrathecally injected with $5 \mathrm{nM}$ DZNep. The intrathecal administration of $40 \mathrm{nM}$ DZNep increased the autonomous activity of BCP rats (see $\underline{\mathrm{S} \text {-Table } 3}$ and $\underline{\mathrm{S} \text {-Figure } 1}$ ). As a result, the application of $20 \mathrm{nM}$ DZNep in this study was reasonable.

Based on our results, an intrathecal injection of DZNep relieved BCP and reduced the levels of EZH2 expression in BCP rats. Similarly, in a rat chronic constriction injury (CCI) model, intrathecal administration of CGRP8-37 (a CGRP antagonist) and GSK126 (an inhibitor of EZH2) attenuated pain hypersensitivity and decreased the levels of EZH2 and H3K27me3 in the spinal dorsal horn. ${ }^{28}$ Yadav et $\mathrm{al}^{8}$ found that an intrathecal injection of DZNep prevents the development of neuropathic pain, and its analgesic effects are associated with suppression of glial activation and the production of TNF$\alpha$, IL-1 $\beta$, and MCP-1. Zhang et $\mathrm{al}^{29}$ reported that EZH2 overexpression abrogates the effects of miR-124-3p on neuroinflammation and neuropathic pain. According to these studies, a potential mechanism of EZH2 in neuropathic pain may be to suppress neuroinflammatory responses. However, BCP includes some common characteristics of inflammatory pain and neuropathic pain. Therefore, one of the underlying mechanisms of EZH2 in BCP may be to suppress neuroinflammation. However, the exact mechanisms must be clarified in models of this condition.

\section{Conclusion}

Taken together, the current study suggests that spinal EZH2 may be involved in the process of bone cancer pain in rats.

\section{Abbreviations}

AS, ambulatory score; BCP, bone cancer pain; DZNep, 3-deazaneplanocin A; EZH2, enhancer of zeste homolog 2; H3K27Tm, trimethylation of 27th lysin of histone H3; PcG, polycomb-group proteins; PRC, polycomb repressive complex; PVDF, polyvinylidene fluoride; PWTpaw withdrawal threshold; RT-qPCR, real-time quantitative PCR; WB, Western blot.

\section{Acknowledgments}

This work was financed by the Kunshan High-level Medical Talents Project (Ksgccrc2004), the Medical Health Science and Technology Project of Kunshan first people's Hospital (CXTD21-C05), and Medical Scientific Research Project of Jiangsu Provincial Health Commission in 2021 (M2021072).

\section{Disclosure}

All authors have no conflicts of interest.

\section{References}

1. Montagnini ML, Zaleon CR. Pharmacological management of cancer pain. J Opioid Manag. 2009;5(2):89-96. doi:10.5055/jom.2009.0010.

2. Paice JA, Mulvey M, Bennett M, et al. AAPT diagnostic criteria for chronic cancer pain conditions. J Pain. 2017;18(3):233-246. doi:10.1016/j.jpain.2016.10.020.

3. Brogan S, Junkins S. Interventional therapies for the management of cancer pain. J Support Oncol. 2010;8(2):52-59.

4. Hébert M, Potin S, Sebbagh M, et al. Rho-ROCK-dependent Ezrin-radixin-moesin phosphorylation regulates Fas-mediated apoptosis in Jurkat cells. $J$ Immunol. 2008;181(9):5963-5973. doi:10.4049/jimmunol.181.9.5963.

5. Cardoso C, Mignon C, Hetet G, et al. The human EZH2 gene: genomic organisation and revised mapping in $7 \mathrm{q} 35$ within the critical region for malignant myeloid disorders. Eur J Hum Genet. 2000;8 (3):174-180. doi:10.1038/sj.ejhg.5200439.

6. Meng XL, Fu P, Wang L, et al. Increased EZH2 levels in anterior cingulate cortex microglia aggravate neuropathic pain by inhibiting autophagy following brachial plexus avulsion in rats. Neurosci Bull. 2020;36(7):793-805. doi:10.1007/s12264-020-00502-w.

7. Yaswen P. HDAC inhibitors conquer Polycomb proteins. Cell Cycle. 2010;9(14):2705. doi:10.4161/cc.9.14.12323

8. Yadav R, Weng HR. EZH2 regulates spinal neuroinflammation in rats with neuropathic pain. Neuroscience. 2017;349:106-1175. doi:10.1016/j.neuroscience.2017.02.041.

9. Zhai M, Yang S, Lin S, et al. Distinct gene expression patterns of ion channels and cytokines in rat primary sensory neurons during development of bone cancer and cancer pain. Front Mol Neurosci. 2021;14:665085. doi:10.3389/fnmol.2021.665085.

10. Yang Y, Li H, Li TT, et al. Delayed activation of spinal microglia contributes to the maintenance of bone cancer pain in female Wistar rats via P2X7 receptor and IL-18. $J$ Neurosci. 2015;35 (20):7950-7963. doi:10.1523/JNEUROSCI.5250-14.2015

11. Diaz-delCastillo M, Hansen RB, Appel CK, et al. Modulation of rat cancer-induced bone pain is independent of spinal microglia activity. Cancers. 2020;12(10):2740. doi:10.3390/cancers 12102740 . 
12. Yang T, Zhou Y, Zhang W, et al. The Spinal $\alpha 7$-Nicotinic Acetylcholine Receptor Contributes to the Maintenance of CancerInduced Bone Pain. J Pain Res. 2021;14:441-452. doi:10.2147/JPR. S286321.

13. Wang LN, Yao M, Yang JP, et al. Cancer-induced bone pain sequentially activates the ERK/MAPK pathway in different cell types in the rat spinal cord. Mol Pain. 2011;7:48. doi:10.1186/1744-8069-7-48.

14. Chen J, Wang L, Zhang Y, et al. P2Y1 purinoceptor inhibition reduces extracellular signal-regulated protein kinase $1 / 2$ phosphorylation in spinal cord and dorsal root ganglia: implications for cancer-induced bone pain. Acta Biochim Biophys Sin (Shanghai). 2012;44(4):367-372. doi:10.1093/abbs/gms007.

15. Hang LH, Xu ZK, Wei SY, et al. Spinal SET7/9 may contribute to the maintenance of cancer-induced bone pain in mice. Clin Exp Pharmacol Physiol. 2017;44(10):1001-1007. doi:10.1111/14401681.12789.

16. Lan LS, Ping YJ, Na WL, et al. Down-regulation of Toll-like receptor 4 gene expression by short interfering RNA attenuates bone cancer pain in a rat model. Mol Pain. 2010;158(4):705-716. doi:10.1186/ 1744-8069-6-2.

17. Vincent K, Wang SF, Laferrière A, et al. Spinal intracellular metabotropic glutamate receptor 5 (mGluR5) contributes to pain and c-fos expression in a rat model of inflammatory pain. Pain. 2017;158 (4):705-716. doi:10.1097/j.pain.0000000000000823.

18. Wang JA, Niu SN, Luo F. Pulsed radiofrequency alleviated neuropathic pain by down-regulating the expression of substance $\mathrm{P}$ in chronic constriction injury rat model. Chin Med J. 2020;133 (2):190-197. doi:10.1097/CM9.0000000000000619.

19. Ji RR, Nackley A, Huh Y, et al. Neuroinflammation and central sensitization in chronic and widespread pain. Anesthesiology. 2018;129(2):343-366. doi:10.1097/ALN.0000000000002130

20. Cao Y, Fan D, Yin Y. Pain mechanism in rheumatoid arthritis: from cytokines to central sensitization. Mediators Inflamm. 2020;2020:2076328. doi:10.1155/2020/2076328.
21. Healy E, Mucha M, Glancy E, et al. PRC2.1 and PRC2.2 synergize to coordinate H3K27 trimethylation. Mol Cell. 2019;76(3):437-452.e6. doi:10.1016/j.molcel.2019.08.012.

22. Lavarone E, Barbieri CM, Pasini D. Dissecting the role of H3K27 acetylation and methylation in PRC2 mediated control of cellular identity. Nat Commun. 2019;10(1):1679. doi:10.1038/s41467-01909624-w.

23. Smit De MH, Duin Van J. Secondary structure of the ribosome binding site determines translational efficiency: a quantitative analysis. Proc Natl Acad Sci U S A. 1990;87(19):7668-7672. doi:10.1073/pnas.87.19.7668.

24. Yan X, Hoek TA, Vale RD, Tanenbaum ME. Dynamics of translation of single mRNA molecules in vivo. Cell. 2016;165(4):976-989. doi:10.1016/j.cell.2016.04.034.

25. Ruchi Y, Xisheng Y, Maixner Dylan W, et al. Blocking the GABA transporter GAT-1 ameliorates spinal GABAergic disinhibition and neuropathic pain induced by paclitaxel. J Neurochem. 2015;133 (6):857-869. doi:10.1111/jnc.13103.

26. Wang J, Huang F, Huang J, et al. Epigenetic analysis of FHL1 tumor suppressor gene in human liver cancer. Oncol Lett. 2017;14 (5):6109-6116. doi:10.3892/ol.2017.6950.

27. Girard N, Bazille C, Lhuissier E, et al. 3-Deazaneplanocin A (DZNep), an inhibitor of the histone methyltransferase EZH2, induces apoptosis and reduces cell migration in chondrosarcoma cells. PLoS One. 2014;9 (5):e98176. doi:10.1371/journal.pone.0098176.

28. An Q, Sun C, Li R, et al. Calcitonin gene-related peptide regulates spinal microglial activation through the histone H3 lysine 27 trimethylation via enhancer of zeste homolog-2 in rats with neuropathic pain. J Neuroinflammation. 2021;18(1):117. doi:10.1186/s12974-02102168-1.

29. Zhang Y, Liu HL, An LJ, et al. miR-124-3p attenuates neuropathic pain induced by chronic sciatic nerve injury in rats via targeting EZH2. J Cell Biochem. 2019;120(4):5747-5755. doi:10.1002/ jcb. 27861
Journal of Pain Research

\section{Publish your work in this journal}

The Journal of Pain Research is an international, peer reviewed, open access, online journal that welcomes laboratory and clinical findings in the fields of pain research and the prevention and management of pain. Original research, reviews, symposium reports, hypothesis formation and commentaries are all considered for publication. The manuscript

\section{Dovepress}

management system is completely online and includes a very quick and fair peer-review system, which is all easy to use. Visit http:// www.dovepress.com/testimonials.php to read real quotes from published authors. 